\title{
Por los desfiladeros del significante. Conversaciones con Freud y Lacan: una mirada psicoanalítica a la educación
}

Elba Meana $(*)$

\begin{abstract}
El objetivo de este trabajo es analizar en un texto concreto la imposibilidad de la comunicación según Lacan; ya que entre lo que el "Sujeto" demanda y lo que el "Otro" responde no existe una respuesta adecuada, por cuanto el "Otro" codificará y devolverá de forma invertida el mensaje contestando desde su propio parecer y bajo el imperio de la significación, que, como dice Lacan, remite a otra indefinidamente.
\end{abstract}

Se analiza la naturaleza del significante lacaniano en una noticia periodística, su conformación y función, sus relaciones con el "querer decir", "el decir", y " lo dicho" (efecto perlocucionario), y sus relaciones con el sujeto mítico de la necesidad, así como la constitución del nudo borromeo (rsi); a partir del grafo 1 de Lacan, tomando como fuente un caso que fue viralizado en las redes, vinculado con la situación de rechazo que generó la actitud de un docente en la provincia de buenos aires al tachar un ejercicio matemático de un niño de siete años, considerándolo incorrecto.

E1 corpus seleccionado hace referencia a una situación pedagógica: el hijo de
Ignacio Bárcena interpretó el enunciado: "los siguientes números" como las cifras sucesivas inmediatas, en vez de las cantidades exactas que aparecían escritas con letras. el maestro, al corregir, trazó una enorme $\mathrm{x}$ en rojo sobre las respuestas del estudiante. el padre del niño decidió compartir lo sucedido en su cuenta de twitter. la RAE expuso su punto de vista, avalando al docente.

Se esbozan en un comienzo algunas consideraciones teóricas (también estarán presentes en las notas al final del documento) vinculadas con el grafo I, y posteriormente se analizan en un corpus recortado los aspectos señalados aquí, en los cuatro primeros renglones iniciales. Se finaliza con algunas reflexiones pedagógicas asociadas a la temática elegida para su desarrollo.

\section{Texto/discurso}

\section{Significante/código/mensaje}

E1 plano de lo textual, que algunos especialistas asimilan a discurso, ese sin sentido que da lugar al sentido, ha merecido no poca tinta en el terreno de la Lingüística ${ }^{1}$. En ocasiones se ha descripto (desde esta 
ciencia) al discurso como un texto sometido a las condiciones de producción (autor) o recepción (destinatarios/lectores), es decir, a los contextos históricos y culturales, sociales, en que ha sido gestado y luego leído, pudiendo corresponder estos dos contextos a épocas muy diferentes.

Saussure $^{2}$ afirmaba que la Lingüística correspondía al orden de la Semiología , como ciencia de los signos en el seno de la vida social y esta estaba incluída dentro de la Psicología. Pues bien, la verdad es que estas disciplinas hallan su fundamento en relación con una teoría del sujeto, ya que, como dice Lacan, no hay sujeto si no hay significante que lo funde. E1 concepto de signo formalizado por el lingüista ginebrino perdura hasta el día de hoy; y el desarrollo de esta teoría ha sido de singular importancia en el desarrollo de las ciencias en general y en la teoría lacaniana en particular, y es por ello que aquí se la retoma. Ahora bien, para Saussure el signo estaba conformado por una imagen conceptual y una imagen acústica, en este orden, y además: la primera por encima de la segunda. Ya se verá cómo Lacan altera el orden de esta fracción y por qué. Y también: por qué razón el desarrollo de Saussure es insuficiente $^{3}$ para explicar la conducta humana, la forma en que el sujeto barrado, o sujeto social, como lo llama Lacan, tramita sus demandas, transformándose en sujeto mítico de la necesidad, una tal que nunca alcanza a ser satisfecha de verdad.

Hay una función significante en Lacan (porosa y en sombra en Saussure), un plus de significado; signado en lo que se dice, más allá de lo que se ha querido decir: el maravilloso y mágico desfiladero del significante: de a poco uno va comprendiendo que es vacío y solo tiene significado en el plano de abajo (el de la conciencia). Y recién entonces uno va comprendiendo el por qué del lenguaje del analista ${ }^{4}$, por qué ese empecinamiento en "no comprender", porque entiende ahora que no debe, no debiera, más que "interpretar", atenerse a lo que dice más allá del "querer decir", abismarse a las ventanas de la ficción para poder ver, como un gran "voyeur", el paisaje de lo real, que podrá ver, una vez que hubiera traspasado los bosques de lo simbólico e imaginario. De esa integración tripartita es que emerge el sujeto en Lacan.

Se podrá ver a lo largo del trabajo, cómo el significante va por sus desfiladeros, como le gusta decir a Lacan, destilando un lenguaje, que en ocasiones se lo llamará "código", en otras "mensaje"; deberá toparse con una "sanción", que habrá de restringirlo; pero al final, de todas maneras, algo caerá al vacío.

Es que el punto del deseo no está en la voluntad, sino en el plano del inconsciente. Y el Inconsciente, repite Lacan, está estructurado COMO un lenguaje ${ }^{5}$, aunque no es el mismo lenguaje, al menos, en el marco que lo estudia la Lingüística.

Es que hay una sobredeterminación del Inconsciente sobre el código, lo rige, lo regula, lo corrige, lo azuza, El código no lo resiste y se cae. Así como se cae el sujeto unario, la totalidad materna en el estadío del espejo, el deseo semi satisfecho también 
cae, para que nazca el sujeto barrado, eje estructurador del pensamiento lacaniano.

No es que el sujeto utilice el significante para comunicarse con el sujeto, es que un significante utiliza un sujeto para comunicarse con otro significante ${ }^{6}$, transita primero por el sendero de lo unario, hasta encontrar un mínimo de simbolización, que le permite transformarse en binario, para que lo real deje de ser real y pase a ser realidad. $Y$ el contraste aparece ${ }^{7}$.

El sujeto es efecto del significante, no es un instrumento. El sujeto es el instrumento, tal como afirma Benveniste ${ }^{8}$.

Pero ocurre que la teoría lacaniana no es un sistema (como es el signo para Saussure) sino una paradoja heurística, porque todas sus construcciones se pueden desmontar, contrariar, ampliar, así como la misma idea de código, que va creciendo y se va complejizando a medida que el lector se va abismando en el conocimiento de los grafos, va comprendiendo el nudo borromeo y sus componentes (RSI).

Lacan no propone, como los generativistas, un sistema arbóreo que represente la mente. Lacan es una topología, una guía para comprender, de forma geométrica $y$ condensada, las cuestiones que plantea sobre la naturaleza humana y su psiquis. De esa paradoja heurística, como se verá, es que nace lo científico en él, al convertirse en la única ciencia que es capaz de alcanzar el límite entre la mirada y la visión.

E1 lenguaje es el primer otro, que viene a través de otro "otro": el Gran Otro (la madre), de cuya mirada precisa, porque en ella está centrado su deseo; que es el "objeto A", heredero del estadío del espejo, en que el uno y la totalidad ${ }^{9}$, y la indiferenciación, era su rasgo singular. Después, cuando supere esa etapa unaria y se transforme en binaria podrá transformarse en objeto a con minúsculas. Pero este relato tiene descenlace trágico: algo del orden del significante, del deseo caerá, y hasta será preciso que caiga, porque así es, y así debe ser.

\section{El niño que transgredió la consigna. Análisis del corpus}

Según el lingüista ginebrino lo que 1lamamos significado está en íntima conexión con lo que pensamos y con lo que sentimos, y ese significado busca el significante oportuno para expresarlo. Es el signo para Saussure.

Los elementos de análisis que son factibles de ser visibilizados a través de la inversión de los términos saussurianos que en cambio propone Lacan configuran una particular descripción. Los componentes que entran en juego en el concepto de significante lacaniano configuran un todo en que las partes constitutivas se van asociando geométricamente, conforman algoritmos, figuras, grafos, que serán sus herramientas pedagógicas, una clave heurística de interpretación.

Para analizar más palmariamente la situación pedagógica que se presenta en el corpus de este trabajo es imprescindible en primer lugar, describir los componentes del Grafo I, y su función.

- En la imagen, delta es el sujeto mitico de la necesidad. Necesidad es lo que se desea y no se tiene. Aquí la dominancia del 


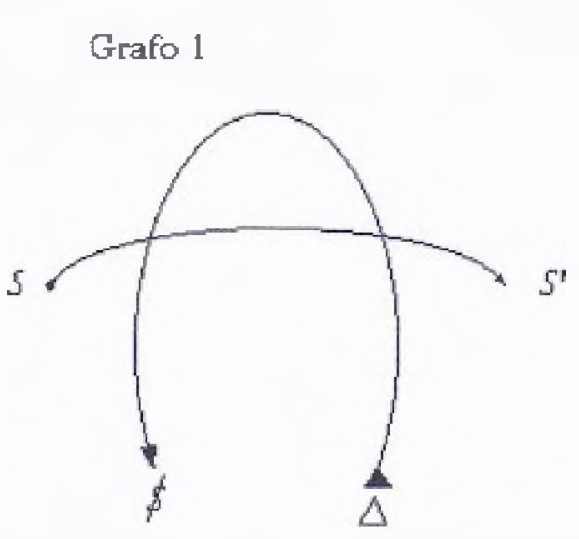

significante es el Inconsciente, y el deseo pasa, como dice Lacan, por los desfiladeros del Inconsciente

- s y s' son diacronía y sincronía ${ }^{10}$, el decir, el lenguaje en sus aspectos filogenéticos (en su evolución a través del tiempo) y ontogenéticos (lo que mueve al sujeto en el plano lingǘstico a comunicarse), aspectos estos que la Linguística ha estudiado ampliamente. Es importante resaltar aquí que para Lacan el lenguaje es autónomo y tiene su propia ley: la que dicta el sistema, la norma y el uso ${ }^{11}$,

- C: Código: tesoro del significante del otro.

- M: Mensaje: significado del otro.

¿Cómo llega al código el sujeto mítico de la necesidad?, ¿y de qué manera y con qué recursos hará uso del lenguaje? Tiene a su disposición un vasto repertorio léxico, el que es propio del lenguaje, de un idioma en particular, con todo su bagaje de múltiples y diversas expresiones.

En este caso la maestra formuló la siguiente consigna:



"Escribe con cifras los siguientes números",

cuando en realidad debió presentado la consigna de la siguiente manera:

"Convertir estos números que están más abajo a texto o letras", ya que la acción de convertir es la verdadera operación que se les proponía.

En verdad el enunciado "los siguientes números", no son números, son BÁSICAMENTE LETRAS que denotan números. Existen solamente dos tipos de símbolos, el numérico y el alfabético, y las actividades de conversión de un tipo de símbolo a otro exige una capacidad de abstracción que a esa edad solamente se puede enseñar ( $y$ aprender) en situaciones muy concretas y con las palabras adecuadas. En el momento de la redacción de la consigna sobrevino la primera sanción del código, en tanto y en cuanto la maestra formuló la consigna de manera inadecuada. $\mathrm{Y}$ esto ocurre porque estas posibles equivocaciones, o incorrecciones también son propias del lenguaje. E1 lenguaje lo permite. Por ejemplo, así como los chinos tienen siete maneras distintas de 
decir "arroz", palabra sagrada y eje de su economía. El código de ellos lo permite.

A ello habría que agregar que, como sostiene Noam Chomsky, los niños tienen una lógica asombrosa para tipificar los actos de habla. Saben cómo se forman los participios pasado y construyen expresiones del tipo de: "morido", "escribido", mostrando de este modo que conocen a la perfección que los verbos regulares forman su participio pasado correspondiente agregando la desinencia: "ado/ido"; porque tienen una gramática incorporada al tiempo en que adquieren el lenguaje.

En este caso el niño no absorbió toda la demanda (surgida de una necesidad $\mathrm{x}$ ) proveniente de su maestro, e interpretó el sintagma: "siguiente" siguiendo la lógica chomskiana (sabemos que el lenguaje para Saussure es arbitrario y no lógico). Este paso correspondería a la segunda sanción del código. Allí el significado, visto desde la óptica del docente se ha caído, al impulsar al alumno a responder fuera del mensaje planteado por el docente.

El mensaje va y regresa, en tanto hecho comunicativo, sancionado, modificado. En este caso, como se dijo, siguiendo la lógica del niño, que interpretó como "siguiente" el número inmeditamente posterior.

Cabe resaltar el efecto que este hecho tuvo entre los que encarnan, podría decirse "la ley", es decir, la voz autorizada del manual.

En el caso puntual que aquí se analiza el rector (en el corpus, es el ejemplo 4) solamente se remitió a observar "la relevancia de las calificaciones", señalando el efecto negativo que la actitud del docente podría tener en el alumno y "su motivación", según palabras del rector.

En lo atinente a la interpretación del niño, dice el rector en el video, sería necesario explicarle al niño el uso de "siguiente" en diferentes contextos.

En el mismo sentido obró la RAE al limitarse a expresar: "lo natural es que se escriban los números que se citan a continuación".

Estos enunciados involucran ACTOS

\section{DE HABLA.}

Y entonces la pregunta que surge a continuación es:

¿Cómo están actuando aquí los que representan a la voz del saber?, ¿será este el discurso universitario o en cambio podría muy bien ejemplificar el tipo del discurso del amo?

Veamos: En cuanto al "agente": el discurso de este docente, el del rector, y el de la RAE es un discurso sin fisuras. El significante es todo el amo, es activo y es pasivo. En cuanto al "goce", se prohíbe el goce, es el lugar de la verdad irrefutable, la que no se cuestiona. En lo atinente "al otro", no existe, el niño no existe, no respondió lo que docente esperaba y fue sancionado sin que nadie analizara en forma autorizada lo que realmente ocurrió. El alumno está obstruido, no tiene deseo, el maestro controla, es la voz del poder.

Finalmente, en cuanto a la "producción", está desde el vamos perdida, ya que el alumno tendrá que hacer lo que el docente disponga.

En el ejemplo que aquí se comenta era 
de prever que tanto el rector como la RAE hubieran podido tener una actitud más cuestionadora y explicativa de lo que realmente ocurrió. No se sabe si por ignorancia u omisión optaron por bajar su martillo avalando a la institución educativa, como es de costumbre.

En síntesis:

S1 (otro) -------> s2 (saber)

$S$ barrado <---------- a (plus de goce)

El lenguaje es la condición del inconsciente y el malentendido (el del docente y el del alumno, ambos dos) no es un accidente sino un hecho de estructura, que ya se encuentra en la naturaleza misma del significante.

Como es sabido los malos entendidos forman parte de toda comunicación, y no hay comunicación en donde el poder no circule de manera perentoria, el poder y la ideología, en tanto el lenguaje es por definición ideológico.

Roland Barthes divide los sociolectos entre aquellos que están "dentro del poder" (como en este caso lo es el discurso de la maestra, del rector, y de la RAE) y aquellos otros que están fuera, o dice él, o "sin poder o dentro del no poder"12, e ia a incluye a la Psicología y a la Literatura en este segundo caso. Afirma que el discurso psicoanalítico no está directamente ligado a una crítica del poder, tal vez sea por esa búsqueda de paridad entre el consultante y el que propicia la consulta. Catherine Millot habla de un pasaje de la infancia a la madurez ${ }^{13}$.

Más allá de todo, sabemos al final que sobre el inconsciente no se manda, y que, como sostiene Millot, "tampoco domina- mos los efectos de la influencia que ejercemos sobre otro ser"14. Y en este terreno no hay pedagogía que lo regule, puesto que entre toda medida pedagógica se ubica ese inmenso río del inconsciente mediando entre ambos.

Algunas reflexiones pedagógicas

Mecanismos inconscientes median a través del lenguaje entre quien enseña y quien aprende, ya que no todo lo que se enseña es aprendido. Hay también una ilusión de control del proceso pedagógico, que por igual razón es imposible de prever.

E1 pedagogo NUNCA se dirige al yo consciente del niño, su rol será siempre indirecto, y es por este motivo que es recomendable promover situaciones problemáticas interesantes, desplazando la enseñanza de estructuras, como en este caso lo vemos (fue un ejercicio aproblemático), al estudio de procesos de estructuración. Los docentes transmiten saberes ya terminados, construidos (por él); y los alumnos llegan a comprender por caminos diferentes, pero este aspecto rara vez se tiene en cuenta.

Castorina habla del niño "asocial", al sostener que quien enseña convierte una diferencia en desigualdad. Sabido es que ambos actores pedagógicos están mediados por el significante, en términos de Lacan; también por el niño que alguna vez fue ese docente, y por las "ilusiones pedagógicas"15 de las que éste parte, entre otras: la de un saber ya construido (difícil de sistematizar por un alumno que al momento de la explicación carece de los esquemas que pue- 


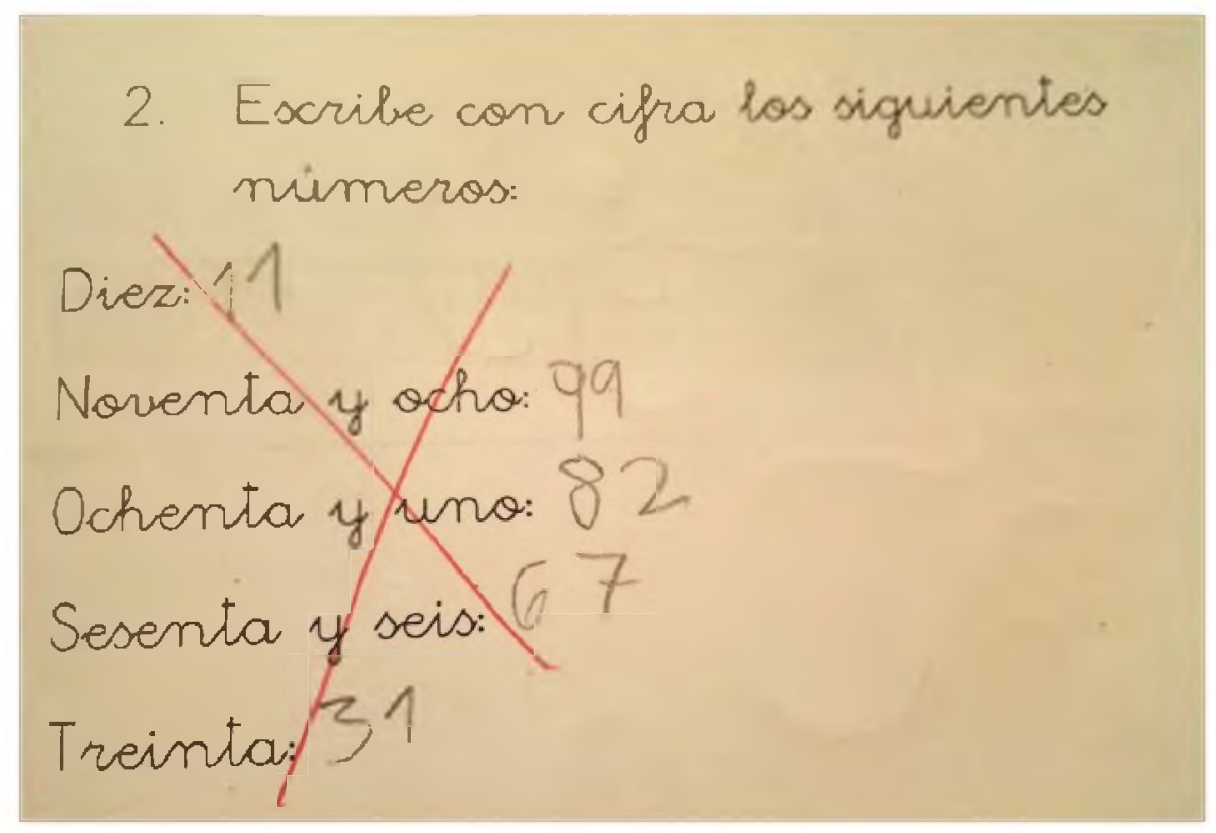

dan facilitar la comprensión de lo que se enseña). Otra ilusión pedagógica es la del "alumno ideal". Éstas y otras idealizaciones juegan un sustantivo papel en el discurso didáctico, que, sin saberlo conscientemente el docente, inciden notablemente en el acto pedagógico. Este es un aspecto muy escasamente difundido, en todos los niveles educativos de la República Argentina.

Una de las ilusiones pedagógicas más singulares que tiene el docente según Castorina es la de que, en el fondo más profundo de su ser, por más cursos de formación profesional que pudiera haber aprobado, terminará enseñanado como él fue enseñado. Y esta imagen que ilustra la página da cuenta de un estilo muy usado antaño: el tan temido tachón, esta actitud no es coetánea a ese docente. Ese tachón es el que él conoció cuando fue alumno.
Es muy elocuente esta imagen. Abismarse en la lectura del maestro Freud es comprobar toda la prodigiosa influencia que éste ejerció en Castorina; la importancia que este último le otorga a la valiosa mediación del lenguaje (y por tanto del inconsciente) entre uno que aprende y otro que enseña desde el niño que fue ha sido un mágico descubrimiento al escribir este trabajo.

Millot sostiene que el deseo del niño se encuentra doblemente alienado, en primer lugar por sus padres, al estar investido de connotaciones desde antes de nacer, y, también, por ese resto irreductible en que se constituye, al existir entre él y ellos, (y 'se agregaría además, entre él y todos sus docentes): ese inextricable DESFILADERO DEL SIGNIFICANTE, como dice el título de este trabajo. Desde el Gran otro (A) al otro (a). 


\section{DEFINICIONES DE TEXTO}

1) Lázaro Carreter: "Todo conjunto analizable de signos"

2) Dubois y otros: "Conjunto de enunciados lingǘsticos sometidos al análisis: el texto es, por tanto, una muestra de comportamiento lingüístico, que puede ser escrito o hablado".

3) Hjelmslev: "Un enunciado cualquiera, hablado o escrito, largo o breve, antiguo o moderno.

4) Rosenthal: "Producto del habla (enunciado) reproducido por escrito".

5) Schmidt: "Todo aquello que es lenguaje, en forma comunicativa o social".

6) Dressler: “... mayor signo lingüístico".

7) Satkov: "Es un Sistema de Enunciados que se entienden como oraciones actualizadas. Es producto de la actividad lingüístico- espiritual del hombre, es una unidad especial de características semánticas y estructurales y cumple funciones comunicativas".

8) Isemberg: "Es la forma primaria de organización en la que se manifiesta el ser humano".

9) Van Dijk: "Concepto abstracto que se manifiesta o realiza en discursos concretos. Se sitúa en el campo de la Semántica y su análisis es abstracto, restrictivo y general, no explica los conocimientos, creencia u otros sistemas cognitivos involucrados ni considera la información general o subjetiva, estudia el significado y la referencia aislados de los actos de habla, de los esquemas superestructurales, de los efectos retóricos, y, consiguientemente, del contexto sociocultural.

10) María Delia Fernández: "Es una Unidad del Lenguaje en uso, no es una entidad gramatical".

11) Lotman y Escuela de Tartu (herederos de Bajtin): "Conjunto sígnico coherente". Un ballet, un espectáculo teatral o una conversación, un cuadro, o una obra literaria pueden constituir un texto, desde esta perspectiva.

12)Lyons:. "Todo pasaje coherente de discurso hablado o escrito, sea conversación o monólogo. Producto restringido al campo verbal. De carácter estructurado"

Según este autor su principal característica (con la que la mayoría de los autores acuerdan) es que es una unidad lingüística comunicativa, que concreta una actividad verbal con carácter social, en que la intención del hablante produce un cierre comunicativo, de modo que el texto es autónomo".

13)Según Enrique Bernárdez, de todas estas definiciones se pueden inferir algunos rasgos comunes:

-Unidad Comunicativa

-Producto de actividad

-Sucesión de Oraciones

-Signo lingǘstico

-Cierre semántico, relaciones internas.

- Un conjunto múltiple de factores considera fundamentales los siguientes criterios:

- A) Carácter comunicativo.

- B) Carácter pragmático

- C) Carácter estructurado: existencia de reglas propias del nivel textual- 
Así, según el mismo Bernárdez, se podrían enunciar las siguientes características:

"Texto es la unidad lingüistica comunicativa fundamental, producto de la actividad verbal humana, que posee siempre carácter social; está caracterizada por su cierre semánti-

1 BERNÁRDEZ, E. (1982): "Introducción a la Lingüística del texto". Espasa Calpe.

2 Saussure, F. (1945). “Curso de Lingüística General”. Buenos Aires, Losada.

3 "La Lingüística, cuyo modelo es el juego combinatorio que opera espontáneamente, por sí solo, de manera presubjetiva, esta estructura le da sus status al inconsciente. En todo caso, ella no asegura que el término inconsciente encierra algo calificable, accesible y objetivable.

4 Lacan, J. (1964):"El Inconsciente Freudianao y el nuestro". En: "Libro 11. "Los cuatro conceptos fundamentales del Psicoanálisis". Capítulo II. p. 28.

4 “E1 Psicoanálisis es ya a estas alturas una ciencia? (...) Me atrevo a enunciar como una verdad que el campo freudiano solo era posible cierto tiempo después de la emergencia del sujeto cartesiano, por el hecho de que la ciencia moderna empieza después del paso inaugural dado por Descartes.

Mucho tiempo después de la revolución cartesiana y de la revolución newtoniana todavía se percibe (...) una teoría religiosa de la tierra como gran fetiche. (...) el análisis de luz permitió ver miles de cosas de los astros. P 155 . co y comunicativo, así como por su coherencia profunda y superficial, debida a la intención (comunicativa) del bablante de crear un texto integro, y a su estructuración mediante dos conjuntos de reglas: las propias del nivel textual y las del Sistema de la Lengua".

Lacan, J. (1964):"El Inconsciente Freudianao y el nuestro". En: "Libro 11. Los cuatro conceptos fundamentales del Psicoanálisis. Capítulo II. p. 155.

5 El Inconsciente está estructurado como lenguaje".

Lacan, J. (1964):"E1 Inconsciente Freudianao y el nuestro". En: "Libro 11. Los cuatro conceptos fundamentales del Psicoanálisis. Capítulo II. p. 155

6 "El sujeto está dividido. Un significante dado (s1) es lo que representa a este sujeto dividido por otro significante. A diferencia del signo que representa algo para alguien, un significante representa a un sujeto para otro significante, no hay de él otra definición, apuntaba Lacan".

-Assoun, Paul-Laurent; (2008) “Lacan”. Buenos Aires: Amorrortu. p. 72.

7 Este estado lo alcanza recién en el tercer tiempo del Edipo.

En el primer tiempo el infans está en situación de arrogación con respecto a su madre.

Ya en el segundo tiempo, el niño debe renunciar a su deseo por su madre; y aparece la sustitución, en la figura del padre. Aquí se gesta el inconsciente y el significante se torna binario. Es el surgimiento de la prohibición del incesto. 
En la misma construcción de la identidad está la ficción, tiene efectos performativos. El sujeto social se construye a través de una imagen fictica; hay allí una hiancia, a través de donde algo (entre lo que se necesita y se consigue) se escapa.

En el tercer tiempo, finalmente, el sujeto es efecto del significante. No es un instrumento. El sujeto lo es.

8 Dice Benveniste: "Hablar de instrumento es oponer hombre y naturaleza. El pico, la flecha, la rueda, no están en la naturaleza. Son fabricaciones. E1 lenguaje está en la naturaleza del hombre, que no la fabricado. (...) Nunca llegamos al hombre separado del lenguaje, (...), es un hablante el que encontramos en el mundo.

Benveniste, E. (1977). "De la subjetividad en el lenguaje2. En: "Problemas de Lingüística General". S XXI editores, Capítulo XV, p. 180.

Según G. Lombardi, a través de Benveniste Lacan había tomado conocimiento de la teoría de los actos de habla (speech acts) de John L. Austin. El golpe mortal de Benveniste a la Lingüística estableciendo la subjetividad del hablante en el hilo del discurso, ha sido enorme, y se descarta que Lacan tenía ese conocimiento.

9 En el estadío del espejo todo lo que desea el niño es el deseo de su madre, $y$ por tanto no tiene significantes. La madre es quien dicta la ley.

El objeto del deseo es el falo de la madre, (podría interpretarse como "el faro"), su reconocimiento. Lo que luego será el "objeto a”, con minúsculas. En esta misma construcción de la identidad está la ficción, y tiene efectos performativos; ya que el sujeto social se construye a través de una imagen ficticia. Toma como totalidad lo que solo es una parte. Esa es la metonimia del deseo. Hay allí una hiancia a través de donde "algo" se escapa: entre lo que se necesita y se consigue.

Este momento del estadío del espejo, del infans hecho uno y todo con su madre, podría compararse a ese tiempo del enamoramiento que Borges define así:

"Enamorarse es producir una mitología privada. Es bacer del universo una alusión a la única persona indudable"

Borges, J. L. (1932). Prólogo al libro de Roberto Godel: "Nacimiento del fuego". Buenos

Aires, Francisco A. Colombo, 1932

10 Los significantes solo pudieron constituirse en la simultaneidad en razón de una estructura muy definida de la diacronía constituyente. La diacronía está orientada por la estructura.

Tanto es así que Freud lo dice sin saber sin que lo dice cincuenta años antes que los lingüistas. Lacan, J. (1964):"El Inconsciente Freudianao y el nuestro". En: “Libro 11. Los cuatro conceptos fundamentales del Psicoanálisis. Capítulo II. p. 54 .

11 Eugenio Coseriu (1921-2202) revisó la dicotomía saussuriana lengua-habla, añadiendo al eje la norma: Habla / Norma / Sistema. La Norma sería lo que en términos de Lacan es la ley, y que en este trabajo está representado en las palabras de la RAE.

12 Barthes, R. (1994). "El susurro del lenguaje”. Bs. As. Paidós. p.128.

13 Millot, C. (1990). "Freud, antipedagogo". Bs As.Paidós, p.166.

14 Millot, C. op. cit, p. 198.

15 Castorina, J /Lenzi, A. y Fernández, S.(2.000) "Psicogénesis e Ilusiones pedagógicas". En: "Psicología genética y los procesos de aprendizaje". Miño y Dávila. p. 156. 







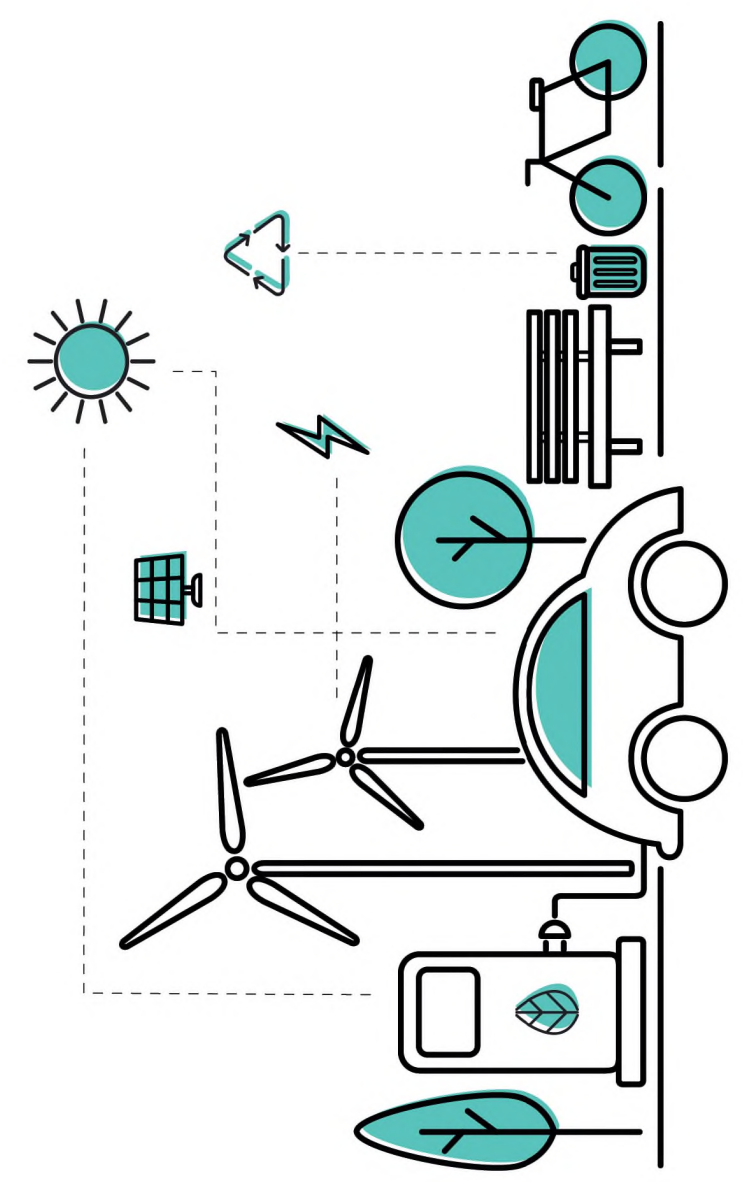


